\title{
Safety behavior and incident experience of worker in gas stations of Suratthani Province, Thailand
}

\begin{abstract}
Transportation development in Thailand has grown at a rapid pace. LPG is a relatively popular automotive fuel in Thailand. The public have more interest in accident, prevention and reduce incidents in the workplace. Therefore, the aims of this research is to study safety behaviors, incident experience (IE) and investigate the safety behavior among worker who have never had incident experience (IE1) and worker who have had incident experiences (IE2) in gas stations. There were 76 respondents. We carried out an exploratory and descriptive study with respondents 19 LPG stations in Suratthani province, Thailand. The majority of workers have had incident experience in LPG stations. The biggest characteristic of these incidents were in process of filling LPG from the disperser to the customer's car. There were leakage from the customer's car and leakage from the equipment in the LPG station. The majority of consequences were the release of gas and collisions resulting in minor, major injury and other results. Besides that, the overall safety behavior of workers was very good. There were some behaviors where the level was moderate and poor. The results showed overall that (IE2) workers had better safety behavior than (IE1) workers. Hence, the companies have to maintain or promote good behaviors. Companies need to provide proper safety training, continually monitor and check to ensure good standards are maintained.
\end{abstract}

Keyword: Incident experience; LPG; Safety behavior 